\title{
Research Article \\ Optimal and Adaptive Virtual Unidirectional Sound Source in Active Noise Control
}

\author{
Dariusz Bismor \\ Institute of Automatic Control, Silesian University of Technology, ul. Akademicka 16, 44-100 Gliwice, Poland \\ Correspondence should be addressed to Dariusz Bismor, dariusz.bismor@polsl.pl
}

Received 21 November 2007; Revised 22 February 2008; Accepted 5 May 2008

Recommended by Marek Pawelczyk

One of the problems concerned with active noise control is the existence of acoustical feedback between the control value ("active" loudspeaker output) and the reference signal. Various experiments show that such feedback can seriously decrease effects of attenuation or even make the whole ANC system unstable. This paper presents a detailed analysis of one of possible approaches allowing to deal with acoustical feedback, namely, virtual unidirectional sound source. With this method, two loudspeakers are used together with control algorithm assuring that the combined behaviour of the pair makes virtual propagation of sound only in one direction. Two different designs are presented for the application of active noise control in an acoustic duct: analytical (leading to fixed controller) and adaptive. The algorithm effectiveness in simulations and real experiments for both solutions is showed, discussed, and compared.

Copyright (C) 2008 Dariusz Bismor. This is an open access article distributed under the Creative Commons Attribution License, which permits unrestricted use, distribution, and reproduction in any medium, provided the original work is properly cited.

\section{INTRODUCTION}

Active noise control is mainly concerned with low-frequency sounds that cannot be suppressed in passive manner at reasonable cost. Other prerequisite for using ANC is that it does not obstruct media flow in ducts, for example, air conditioning ducts. Unfortunately, almost all duct applications have acoustic feedback; a phenomenon that can heavily decrease or even destroy the results of otherwise properly setup control system $[1,2]$.

There are several methods for avoiding acoustic feedback in duct applications [2-6]. The best one that avoids acoustic feedback completely is to use nonacoustic reference sensor. Unfortunately, this usually limits the application to cancel the tonal sounds and does not allow for the reduction of broadband noise. Another approach to acoustic feedback is to internally process signal from the reference microphone in a way allowing to compensate for this effect $[2,4-6]$. This technique, usually called "feedback neutralisation," uses a model of acoustic feedback path to filter the controller output and then subtract it from the reference signal. In the simplest case, the model is obtained offline and does not account for time variation of the feedback path. Moreover, the method requires a model of the acoustic feedback path and therefore is computationally more expensive.

The approach to acoustic feedback cancellation presented in this paper is called virtual unidirectional sound source (VUSS). The method, in authors opinion, has some advantages over well-known two loudspeaker active noise control system in duct applications. First, it clearly separates the subsystem responsible for neutralisation of undesired feedback from active noise control part. Second, its adaptive version can also be applied in case of varying acoustic path responses. Last, virtual unidirectional sound source can also be applied in more general active control of sound applications, not only in active noise control. The paper attempts to explain the theory behind VUSS as well as to give some results showing its excellent performance in case of ANC.

The paper is organised as follows. Section 2 summarises physical basics necessary to understand principles of VUSS operation and its advantage over one-loudspeaker applications. In Section 3, the idea of VUSS is explained together with the original detailed analysis of optimal (fixed) design (Section 3.1) and adaptive approach (Section 3.2). Finally, Section 4 gives the results of simulations and real 


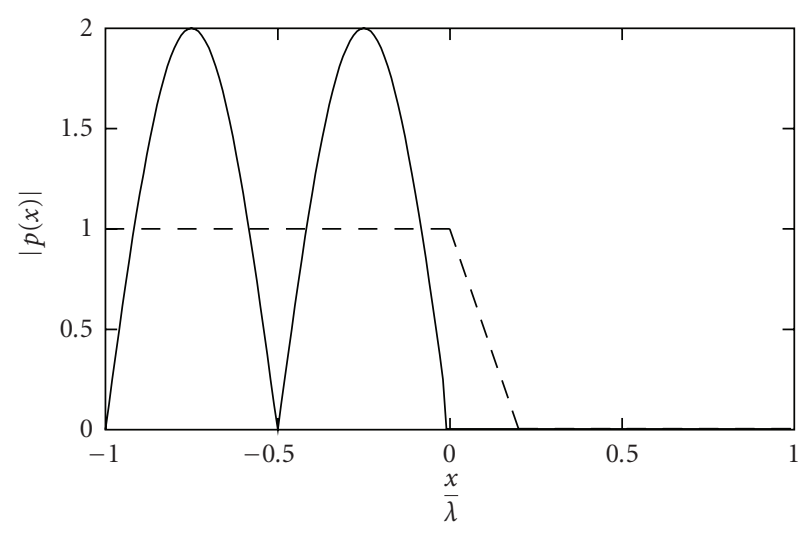

Figure 1: Acoustic pressure distribution in duct: one loudspeaker case (solid) and two loudspeaker case (dashed).

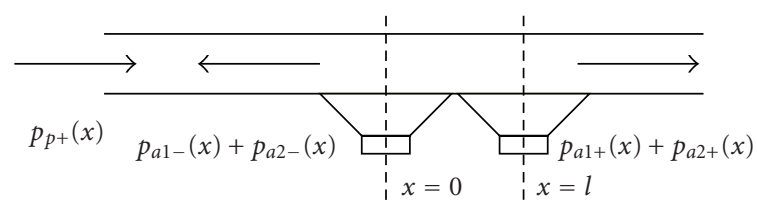

Figure 2: Acoustic duct with two active loudspeakers.

experiments of feedforward active noise control system using virtual unidirectional sound source.

\section{ACOUSTICAL PHENOMENA IN DUCTS}

The simplest solution to acoustic feedback cancellation problem in case where omnidirectional microphone must be used as the reference sensor is called feedback neutralisation $[2,4-6]$. With this approach, only one loudspeaker is used, but the signal obtained from reference microphone is processed in the way allowing for compensation of its influence. If no compensation is performed, severe problems can result. In the simplest case of attenuation of one tone, the pressure distribution upstream secondary source [3] takes shape of standing wave, presented with solid line on Figure 1. If, for particular frequency of this tone, the reference microphone is placed in standing wave nodal point, it will give measurement equal to zero regardless of waves' actual magnitude.

Another situation occurs when two loudspeakers are used, as presented in Figure 2. Assume first loudspeaker is placed in the duct at position $x=0$ and the second at position $x=l$. Denote $p_{p+}(x)$ as the complex pressure [3] at location $x$ produced by primary source emitting tonal sound:

$$
p_{p+}(x)=A e^{-\mathrm{i} k x},
$$

where $A$ is the complex amplitude of the sound wave and where acoustic wave number $k$ is equal to $k=\omega / c_{0}$, with $\omega$ being wave angular frequency and $c_{0}$ being the sound speed in air.

Assume the two loudspeakers on Figure 2 produce sound waves propagating upstream the duct, marked as $p_{a 1-}(x)$ and $p_{a 2-}(x)$, and sound waves propagating downstream the duct, marked as $p_{a 1+}(x)$ and $p_{a 2+}(x)$. Assume also that the sound wave produced by the primary source is propagating downstream the duct, as marked on the figure.

Assume that the complex pressure of sound wave generated by first "active" loudspeaker is given by

$$
\begin{aligned}
& p_{a 1+}(x)=B e^{-i k x} \quad \text { for } x>0, \\
& p_{a 1-}(x)=B e^{i k x} \quad \text { for } x<0,
\end{aligned}
$$

and the complex pressure of sound wave produced by the second "active" loudspeaker is given by

$$
\begin{aligned}
& p_{a 2+}(x)=C e^{-i k(x-l)} \quad \text { for } x>l, \\
& p_{a 2-}(x)=C e^{i k(x-l)} \quad \text { for } x<l,
\end{aligned}
$$

where $B$ and $C$ are the complex amplitudes of the sound waves.

In the situation described above it is possible to set another condition on the system regardless of primary sound source attenuation requirement. In the case of unidirectional sound source, it may be the requirement of self-cancellation of the secondary sound waves propagating upstream the duct:

$$
p_{a 1-}(x)+p_{a 2-}(x)=0 \quad \text { for } x<0 .
$$

In this case, the secondary sources have to be driven in the way providing [3]

$$
B=-C e^{-i k l} .
$$

In the same time to achieve the main ANC goal, which is to cancel the primary sound downstream the second loudspeaker completely, it is necessary to set the complex amplitude:

$$
C=\frac{-A}{2 i \sin (k l)} .
$$

The acoustic pressure level distribution for two loudspeakers case has been shown with dashed line on Figure 1. The figure shows that the sound pressure has been perfectly cancelled at coordinates $x>l$ while the absolute value of the pressure level has not been affected by the secondary sources at negative coordinates $x$. The zone between the secondary loudspeakers $(0<x<l)$ is a transient zone.

Although two-loudspeaker system offers significant improvement over one loudspeaker system, it has its drawbacks too. Specifically, the above control law says that for some frequencies for which $\sin (k l)$ is close to zero, both the secondary sources will have to produce sound waves with amplitude very large, compared with the amplitude $A$ of the primary waveform. Consider, for example, the case where the loudspeakers are separated by the distance $l=0.3 \mathrm{~m}$. In this case the sound waves of frequency $570 \mathrm{~Hz}$ and all its multiplies will result in $\sin (k l)$ equal to, or close to, zero, depending on the actual speed of sound, and thus on temperature, humidity, and so on. This fact will certainly influence the performance of ANC system trying to attenuate such sound waves (see, e.g., the drop in attenuation just below $600 \mathrm{~Hz}$ on Figure 10). 


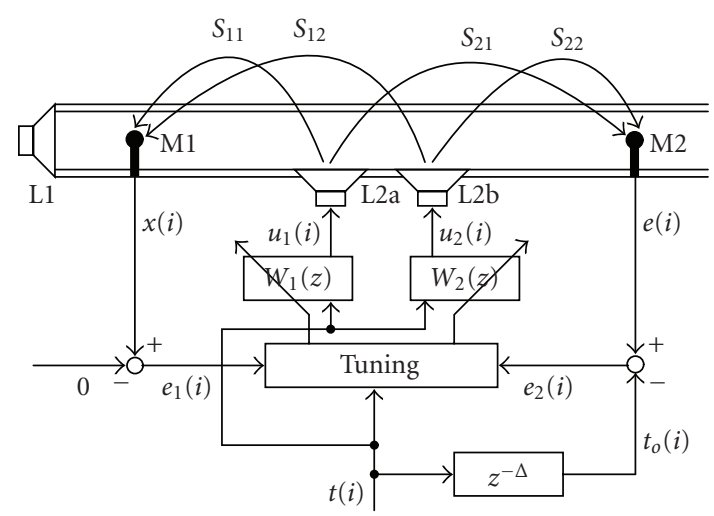

FIGURE 3: Block diagram of virtual unidirectional sound source.

\section{VIRTUAL UNIDIRECTIONAL SOUND SOURCE}

It is well known that introducing the second "active" loudspeaker allows to deal with many one-loudspeaker systems difficulties [4]. Moreover, the analysis outlined in Section 2 proves that it allows also for (hypothetically) perfect cancellation of the acoustic feedback. One of possible systems using two secondary loudspeakers is called virtual unidirectional sound source.

The idea of virtual unidirectional sound source (VUSS) is to use digital signal processing algorithm to drive two loudspeakers in such way that the sound produced by them propagates only downstream the duct [7]. In fact, although the sound generated by each loudspeaker propagates in both directions, the processing algorithm tries to assure that the sound waves propagating upstream the duct are actively cancelled by themselves while those propagating downstream the duct are amplified. The additional advantage of this approach is that it is sometimes possible to equalise the amplitude spectrum of the secondary path transfer function (transfer function between signals $t(i)$ and $e(i)$ on Figure 3 ) that plays very important role in active control of sound [4].

The block diagram of VUSS has been shown in Figure 3. The system contains two "active" loudspeakers, L2a and L2b, as well as two microphones: M1, generally called reference microphone, and M2 called error microphone (the names of the microphones correspond to the role they play in active noise control). The signal to be processed by VUSS is the set point value (or antinoise signal) $t(i)$ produced by ANC algorithm. It is filtered by two filters with transfer functions, $W_{1}(z)$ and $W_{2}(z)$, and sent to the loudspeakers, L2a and L2b, respectively. The error signals are obtained by comparing values acquired from the microphones M1 and M2 with their required values (set points). As the goal of VUSS system is to cancel the influence of the set point value $t(i)$ at the microphone M1 point, the signal $x(i)$ is compared with zero $\left(e_{1}(i)=x(i)-0=x(i)\right)$. Similarly, as the desired signal at the microphone M2 point is the delayed set point value, the second error signal is calculated as $e_{2}(i)=e(i)-$ $t_{o}(i)$. The need for delaying the set point value $t(i)$ comes from condition on causality of the system: no filtration can compensate for the delay time introduced by the acoustic path between the loudspeakers and the M2 microphone. The value of the delay ensuring causality of the algorithm should be chosen before running the algorithm. The error signals $e_{1}(i)$ and $e_{2}(i)$ are used by adaptive algorithm to update parameters of the transfer functions $W_{1}(z)$ and $W_{2}(z)$ (in case of optimal design they are used only for observing the performance of the system).

\subsection{Optimal and suboptimal filter designs}

In this subsection, an attempt to derive analytical optimal and suboptimal filter formulae will be made. The need for suboptimal formula will be explained and feasibility of both the solutions will be discussed.

\subsubsection{Formulation using transfer functions}

Suppose that the whole system presented in Figure 3 is linear and the signal to be cancelled is deterministic, or random wide-sense stationary [8]. Suppose that $W_{1}$ and $W_{2}$ are infinite impulse response (IIR) filters. Figure 3 shows that the control values $u_{1}(i)$ and $u_{2}(i)$ can be expressed as a filtration of set point value $t(i)$ by the $W_{1}$ and $W_{2}$ filters. Using the notation of difference equation, this filtration can be expressed as

$$
\begin{aligned}
u_{1}(i) & =W_{1}(z) \cdot t(i)=w_{1,0} t(i)+w_{1,1} t(i-1)+\cdots \\
& =\sum_{n=0}^{\infty} w_{1, n} t(i-n), \\
u_{2}(i) & =W_{2}(z) \cdot t(i)=w_{2,0} t(i)+w_{2,1} t(i-1)+\cdots \\
& =\sum_{n=0}^{\infty} w_{2, n} t(i-n),
\end{aligned}
$$

where

$$
\begin{aligned}
& W_{1}(z)=w_{1,0}+w_{1,1} z^{-1}+w_{1,2} z^{-2}+\cdots \\
& W_{2}(z)=w_{2,0}+w_{2,1} z^{-1}+w_{2,2} z^{-2}+\cdots
\end{aligned}
$$

In the above equation, $w_{1, n}$ and $w_{2, n}$ are $n$th elements of the $W_{1}$ and $W_{2}$ filter impulse response functions, and are also called filter coefficients. Using this notation, the $z^{-1}$ is treated as discrete shift operator so that $z^{-n} t(i)=t(i-n)$.

From Figure 3 it also follows that the reference signal $x(i)$ due to the secondary sources measured by the M1 microphone can be expressed with the following difference equation:

$$
x(i)=S_{11}(z) \cdot u_{1}(i)+S_{12}(z) \cdot u_{2}(i),
$$

where $S_{11}(z)$ and $S_{12}(z)$ are impulse response functions of electroacoustical paths between the reference signal $x(i)$ and the $u_{1}(i), u_{2}(i)$ control values, including amplifiers, filters, loudspeakers, and others.

Similarly, the signal $e(i)$ measured by the microphone M2 due to the secondary sources can be given by

$$
e(i)=S_{21}(z) \cdot u_{1}(i)+S_{22}(z) \cdot u_{2}(i) .
$$


Considering the above and the definitions of error signals given in the previous subsection, the error signals can be expressed as

$$
\begin{aligned}
& e_{1}(i)=S_{11} \cdot u_{1}(i)+S_{12} \cdot u_{2}(i), \\
& e_{2}(i)=S_{21} \cdot u_{1}(i)+S_{22} \cdot u_{2}(i)-t_{o}(i),
\end{aligned}
$$

where the dependence of $S_{n n}$ transfer functions on $z$ has been omitted to simplify the notation.

The goal of the tuning algorithm is to drive error signals to zero:

$$
e_{1}(i)=0, \quad e_{2}(i)=0
$$

Substituting (7) and (11) into (12), recognising that $t_{o}(i)=z^{-\Delta} t(i)$ gives for all $t(i) \neq 0$

$$
\begin{aligned}
& S_{11} \cdot W_{1}(z)+S_{12} \cdot W_{2}(z)=0, \\
& S_{21} \cdot W_{1}(z)+S_{22} \cdot W_{2}(z)=z^{-\Delta},
\end{aligned}
$$

where $\Delta$ samples delay time was introduced to assure causality of the system.

Solving the above equation set gives the transfer functions of optimal filters:

$$
\begin{aligned}
& W_{\text {lopt }}(z)=-z^{-\Delta} \frac{S_{12}}{S_{11} \cdot S_{22}-S_{12} \cdot S_{21}}, \\
& W_{2 \mathrm{opt}}(z)=z^{-\Delta} \frac{S_{11}}{S_{11} \cdot S_{22}-S_{12} \cdot S_{21}} .
\end{aligned}
$$

Unfortunately, (14) cannot be used in practise due to nonminimumphase nature of transfer functions $S_{n n}$ leading to instability of VUSS filters. Moreover, even when all the transfer functions $S_{n n}$ are minimumphase, the subtraction in denominator of (14) can still result in instability of the whole filter. This is referred to in the literature as "unconstrained controller" [3].

The suboptimal design of virtual unidirectional sound source can be performed after omitting the second condition from (12) set in mathematical derivations. In consequence, it means we do not expect the transfer function from set point value $t(i)$ to microphone M2 signal $e(i)$ to be pure delay, but we allow it to take more complex form. Furthermore, it means that the amplitude spectrum of this transfer function will not be flat over the frequency range under consideration. However, we still request cancellation of the secondary sources influence on the reference signal $x(i)$.

With control goal stated above, there are two equivalent solutions that will be called suboptimal, namely,

$$
\begin{aligned}
& W_{1 \text { sub }}(z)=-S_{12}(z), \\
& W_{2 \text { sub }}(z)=S_{11}(z),
\end{aligned}
$$

or

$$
\begin{aligned}
& W_{1 \text { sub }}(z)=S_{12}(z), \\
& W_{2 \text { sub }}(z)=-S_{11}(z) .
\end{aligned}
$$

Moreover, assuming the $S_{n n}$ transfer functions are of FIR type, the suboptimal solutions are also FIR type.

It is, however, important to notice that in case of suboptimal solution the secondary path transfer function (transfer function between signals $t(i)$ and $e(i)$ on Figure 3 ) is given by

$$
\begin{aligned}
& S(z)=-S_{12} \cdot S_{21}+S_{11} \cdot S_{22}, \text { or } \\
& S(z)=S_{12} \cdot S_{21}-S_{11} \cdot S_{22} .
\end{aligned}
$$

(Again, the dependence of $S_{n n}$ transfer functions on $z$ has been omitted to clarify the notation.) This shows that in case of no demand for equalisation of the secondary path transfer function amplitude spectrum it can assume arbitrary shape, potentially "worse" than in case of single secondary source. This effect must be taken into account when estimating secondary path models required by active noise control algorithm (e.g., by using longer filters).

\subsubsection{Formulation using reference signal matrix}

The following subsection presents result obtained using the approach presented in $[3,9]$, which is based on impulse response of appropriate electroacoustic paths and on the use of filtered-reference signals. Using this approach, the $l$ th error signal ( $l=\{1,2\}$ in this case) can be expressed as a sum of desired signals and contributions from $m$ secondary sources (with $m=2$ in this case) as:

$$
e_{l}(i)=-d_{l}(i)+\sum_{m=1}^{2} \sum_{j=0}^{J-1} s_{l m}(j) u_{m}(i-j),
$$

where $s_{l m}(j)$ is the $j$ th coefficient of impulse response of electroacoustic path between the $l$ th sensor and the $m$ th actuator and $d_{l}(i)$ is desired value of the $l$ th sensor signal. The length of impulse response can be arbitrary number $J$ guaranteeing desired accuracy.

In the same manner, the signals driving actuators can be expressed as

$$
u_{m}(i)=\sum_{n=0}^{N-1} w_{m, n} t(i-n)
$$

where $w_{m, n}$ is the $n$th parameter of the $N$ th order $w_{m}$ FIR filter impulse response.

Substituting (19) into (18) yields

$$
e_{l}(i)=-d_{l}(i)+\sum_{m=1}^{2} \sum_{j=0}^{J-1} \sum_{n=0}^{N-1} s_{l m}(j) w_{m, n} t(i-j-n) .
$$

To derive a matrix formulation for the above equation it is necessary to reorganise the order of filtration of the set point value $t(i)$. To do this, it is necessary to assume that the filters $s_{l m}$ and $w_{m, n}$ are time invariant. If the filtered reference signal is defined as

$$
r_{l m}(i)=\sum_{j=0}^{J-1} s_{l m}(j) t(i-j)
$$


the error signals can be written as

$$
e_{l}(i)=-d_{l}(i)+\sum_{m=1}^{2} \sum_{n=0}^{N-1} w_{m, n} r_{l m}(i-n) .
$$

After defining

$$
\mathbf{w}(i)=\left[\begin{array}{l}
w_{1, i} \\
w_{2, i}
\end{array}\right], \quad \mathbf{r}_{l}(i)=\left[\begin{array}{l}
r_{l 1}(i) \\
r_{l 2}(i)
\end{array}\right] .
$$

Equation (22) can be expressed as

$$
e_{l}(i)=-d_{l}(i)+\sum_{n=0}^{N-1} \mathbf{w}^{T}(n) \mathbf{r}_{l}(i-n) .
$$

Finally, the vector of two error signals can be defined as

$$
\mathbf{e}(i)=\left[e_{1}(i) e_{2}(i)\right]^{T}
$$

and the vector of two desired signals can be defined as

$$
\mathbf{d}(i)=\left[\begin{array}{ll}
d_{1}(i) & d_{2}(i)
\end{array}\right]^{T},
$$

leading to the conclusive formula

$$
\mathbf{e}(i)=-\mathbf{d}(i)+\mathbf{R}(i) \mathbf{W},
$$

where

$$
\mathbf{R}(i)=\left[\begin{array}{llll}
\mathbf{r}_{1}^{T}(i) & \mathbf{r}_{1}^{T}(i-1) & \cdots & \mathbf{r}_{1}^{T}(i-N+1) \\
\mathbf{r}_{2}^{T}(i) & \mathbf{r}_{2}^{T}(i-1) & \cdots & \mathbf{r}_{2}^{T}(i-N+1)
\end{array}\right]
$$

is called matrix of filtered reference signals, and

$$
\mathbf{W}=\left[\begin{array}{llll}
\mathbf{w}^{T}(0) & \mathbf{w}^{T}(1) & \ldots & \mathbf{w}^{T}(N-1)
\end{array}\right]^{T}
$$

is the vector containing all the coefficients of both $W_{1}$ and $W_{2}$ filters (the filters are time independent).

It is interesting to notice that in case of virtual unidirectional sound source the vector of desired signals can be instantiated as

$$
\mathbf{d}(i)=\left[\begin{array}{c}
0 \\
t(i-\Delta)
\end{array}\right] .
$$

Following the methodology presented in [9] the optimal filter solution can be expressed as

$$
\mathbf{W}_{\mathrm{opt}}=\left[E\left\{\mathbf{R}^{T}(i) \mathbf{R}(i)\right\}\right]^{-1} E\left\{\mathbf{R}^{T}(i) \mathbf{d}(i)\right\},
$$

where $E\{\cdot\}$ denotes expectation operator.

The matrix to be inverted is of dimension $N \times N$, where $N$ is the filter length. Fortunately, it can be proved that the matrix is block Toeplitz matrix [3], so effective iterative methods can be applied for inversion. The expectation operator tells that statistical properties of filtered set point value $t(i)$ will be taken into account. The expression under the right-hand side expectation operator, in the above equation, takes particularly the simple form of

$\mathbf{R}^{T}(i) \mathbf{d}(i)=\left[\begin{array}{llll}\mathbf{r}_{2}^{T}(i) & \mathbf{r}_{2}^{T}(i-1) & \cdots & \mathbf{r}_{2}^{T}(i-N+1)\end{array}\right]^{T} t(i-\Delta)$.

\subsubsection{Formulation in frequency domain}

Applying the Fourier transform to (7) and (11) and substituting the transformed (7) into (11) yields

$$
\begin{aligned}
E_{1}\left(e^{i \omega T_{p}}\right)= & S_{11}\left(e^{i \omega T_{p}}\right) W_{1}\left(e^{i \omega T_{p}}\right) T\left(e^{i \omega T_{p}}\right) \\
& +S_{12}\left(e^{i \omega T_{p}}\right) W_{2}\left(e^{i \omega T_{p}}\right) T\left(e^{i \omega T_{p}}\right) \\
E_{2}\left(e^{i \omega T_{p}}\right)= & S_{21}\left(e^{i \omega T_{p}}\right) W_{1}\left(e^{i \omega T_{p}}\right) T\left(e^{i \omega T_{p}}\right) \\
& +S_{22}\left(e^{i \omega T_{p}}\right) W_{2}\left(e^{i \omega T_{p}}\right) T\left(e^{i \omega T_{p}}\right) \\
& -T_{o}\left(e^{i \omega T_{p}}\right)
\end{aligned}
$$

with $z=e^{i \omega T_{p}}$, where $T_{p}$ is a sampling period.

The above equations can be expressed in more convenient matrix form as

$$
\mathbf{e}\left(e^{i \omega T_{p}}\right)=-\mathbf{d}\left(e^{i \omega T_{p}}\right)+\mathbf{S}\left(e^{i \omega T_{p}}\right) \mathbf{W}\left(e^{i \omega T_{p}}\right) T\left(e^{i \omega T_{p}}\right),
$$

where $\mathbf{e}\left(e^{i \omega T_{p}}\right), \mathbf{d}\left(e^{i \omega T_{p}}\right)$, and $\mathbf{W}\left(e^{i \omega T_{p}}\right)$ are transformed vectors defined by (25), (30), and (29), respectively, and the matrix of transfer functions is defined as

$$
\mathbf{S}\left(e^{j \omega T_{p}}\right)=\left[\begin{array}{ll}
S_{11}\left(e^{i \omega T_{p}}\right) & S_{12}\left(e^{i \omega T_{p}}\right) \\
S_{21}\left(e^{i \omega T_{p}}\right) & S_{22}\left(e^{i \omega T_{p}}\right)
\end{array}\right] .
$$

The unconstrained controller can be found by minimising the cost function equal to expectation of the sum of squared errors, independently at each frequency [3]:

$$
J=E\left\{\mathbf{e}^{H} \mathbf{e}\right\}=\operatorname{trace} E\left\{\mathbf{e} \mathbf{e}^{H}\right\} .
$$

Thus, the optimal controller is equal to

$$
\mathbf{W}_{\mathrm{opt}}\left(e^{i \omega T_{p}}\right)=\mathbf{S}^{-1}\left(e^{i \omega T_{p}}\right) \mathbf{S}_{t d}\left(e^{i \omega T_{p}}\right) \mathbf{S}_{t t}^{-1}\left(e^{i \omega T_{p}}\right),
$$

where matrices of power and cross spectral densities are defined as

$$
\begin{aligned}
& \mathbf{S}_{t d}\left(e^{i \omega T_{p}}\right)=E\left\{\mathbf{d}\left(e^{i \omega T_{p}}\right) T^{*}\left(e^{i \omega T_{p}}\right)\right\}, \\
& \mathbf{S}_{t t}\left(e^{i \omega T_{p}}\right)=E\left\{T\left(e^{i \omega T_{p}}\right) T^{*}\left(e^{i \omega T_{p}}\right)\right\} .
\end{aligned}
$$

Assume now that the set point value $t(i)$ used during VUSS learning phase is a sequence of white noise with expected value $\sigma^{2}$. The power spectral density matrix $\mathbf{S}_{t t}$ is then equal to

$$
\mathbf{S}_{t t}\left(e^{i \omega T_{p}}\right)=\sigma^{2}
$$

independent on $\omega$, and the cross spectral density matrix $\mathbf{S}_{t d}$ is equal to

$$
\mathbf{S}_{t d}\left(e^{i \omega T_{p}}\right)=\left[\begin{array}{c}
0 \\
e^{-i \omega T_{p} \Delta} \sigma^{2}
\end{array}\right]
$$

After evaluating inverse of transfer function block matrix $\mathbf{S}\left(e^{i \omega T_{p}}\right)$, the optimal controller obtained with white noise excitation becomes

$$
\mathbf{W}_{\mathrm{opt}}\left(e^{i \omega T_{p}}\right)=\frac{e^{-i \omega T_{p} \Delta}}{S_{11} S_{22}-S_{12} S_{21}}\left[\begin{array}{c}
-S_{12} \\
S_{11}
\end{array}\right],
$$


where the dependence of $S_{n n}$ transfer function on $e^{i \omega T_{p}}$ has been omitted to clarify the notation.

The above equation is consistent with the result obtained in Section 3.1.1. It is, however, still the unconstrained form of the controller which is not feasible for practical implementation.

\subsection{Adaptive filter solution}

This subsection presents an alternative approach to optimal and suboptimal filter designs discussed above. Now we will try to develop an adaptive algorithm with the goal defined by set of (12). We will assume that $W_{1}(z)$ and $W_{2}(z)$ are in form of finite impulse response filters so the filter coefficients can be stored in a vector similar to this defined by (29), but now with coefficients varying slowly (compared to timescales of plant dynamics) in time.

Although there are many algorithms that can be used to tune filter coefficients [4], two-channel filtered-x LMS algorithm was chosen for the following derivation and experiments. Its advantages are simplicity and robustness, even if speed of convergence is not the best. Using twochannel FXLMS algorithm, the update equation is given by

$$
\mathbf{W}(i+1)=\mathbf{W}(i)+\mu \mathbf{R}^{T}(i) \mathbf{e}(i)
$$

where $\mu$ is step size and $\mathbf{R}(i)$ and $\mathbf{e}(i)$ are defined by (28) and (25), respectively.

The matrix of reference signals $\mathbf{R}(i)$ is generated by filtration of the set point value by electroacoustic path transfer functions $S_{11}(z)$ to $S_{22}(z)$. In practise, the latter can be only estimated yielding a matrix of estimated plant responses $\hat{\mathbf{R}}(i)$. The estimation must take place before running FXLMS algorithm and is usually done by separate identification procedure. The same procedure can also be turned on during active noise control phase after detecting substantial changes in plant dynamics (e.g., changes in air temperature).

To study convergence properties of the above algorithm, it is necessary to substitute (27) into (42) with estimated plant responses matrix, producing

$$
\mathbf{W}(i+1)=\mathbf{W}(i)+\mu\left[-\hat{\mathbf{R}}^{T}(i) \mathbf{d}(i)+\hat{\mathbf{R}}^{T}(i) \mathbf{R}(i) \mathbf{W}(i)\right] .
$$

If the adaptive algorithm is stable, it will converge to the solution setting the expectation value of the term in bracket to zero [3]. The steady-state vector of filter coefficients will therefore be equal to

$$
\mathbf{W}_{\infty}=\{E[\hat{\mathbf{R}}(i) \mathbf{R}(i)]\}^{-1} E[\widehat{\mathbf{R}}(i) \mathbf{d}(i)]
$$

The above result for the adaptive algorithm steady state is in coincidence with the optimal solution presented in Section 3.1.2 (31) if and only if the estimated matrix of reference signals $\widehat{\mathbf{R}}$ is equal to the true matrix of reference signal. This validates, however, the methodology.

The precise consequences of nonperfect matching of true plant responses contained in the matrix of reference signals on adaptation process are still unknown. The strongest

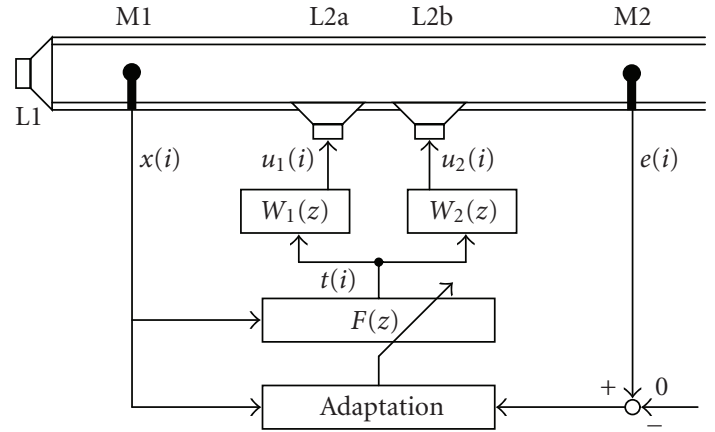

FIGURE 4: Active noise control system using virtual unidirectional sound source.

result has been shown by Wang and Ren [10], the sufficient condition for stability of adaptation. Nevertheless it is not a necessary condition and it has been obtained with the small step size assumption.

During this research it was useful to introduce an additional weight parameter $\beta \in(0,1)$ to specify which of the goals defined in (12) should have more bearing on adaptation process. For such case the definition of error vector $\mathbf{e}(i)$ was modified as follows:

$$
\mathbf{e}^{\prime}(i)=\left[\begin{array}{c}
(1-\beta) e_{1}(i) \\
\beta e_{2}(i)
\end{array}\right]=\left[\begin{array}{cc}
(1-\beta) & 0 \\
0 & \beta
\end{array}\right] \mathbf{e}(i)=\mathbf{B e}(i)
$$

When $\beta$ is close to zero, the algorithm is better excited along the modes responsible for neutralisation of the acoustic feedback effect, while when $\beta$ is close to one the algorithm puts more stress on equalising the secondary path transfer function.

Introducing the weight parameter $\beta$ modifies the steadystate vector of filter coefficients giving

$$
\mathbf{W}_{\infty}=\{E[\hat{\mathbf{R}}(i) \mathbf{B R}(i)]\}^{-1} E[\hat{\mathbf{R}}(i) \mathbf{B d}(i)]
$$

The maximum step size parameter, $\mu$, assuring convergence of the whole algorithm is given by

$$
0<\mu<\frac{2 \operatorname{Re}\left(\lambda_{\max }\right)}{\left|\lambda_{\max }\right|^{2}}
$$

where $\lambda_{\max }$ denotes maximum eigenvalue of $\hat{\mathbf{R}}(i) \mathbf{B R}(i)$ matrix rather than $\hat{\mathbf{R}}(i) \mathbf{R}(i)$ as was in the original solution [3]. The weight parameter $\beta$ can therefore influence, to some degree, the eigenvalues of the matrix under consideration.

\section{ACTIVE NOISE CONTROL WITH VUSS}

\subsection{Active noise control algorithms}

Feedforward active noise control system using virtual unidirectional sound source has been shown in Figure 4. This system uses a finite impulse response filter as control filter with different adaptation algorithms. As in case of all feedforward algorithms, only the reference value $x(i)$ is used 
by the control filter $F(z)$ to produce the set point value $t(i)$. The set point value is then processed by VUSS filters $W_{1}(z)$ and $W_{2}(z)$ to give two control values $u_{1}(i)$ and $u_{2}(i)$ that are amplified and sent to the loudspeakers (via amplifiers and reconstruction filters not shown on the figure). The adaptation algorithm on the other hand uses the reference signal $x(i)$ as well as the error signal $e(i)$ to tune the control filter coefficients.

The duct used in experiments described below was made out of wood. It was $4 \mathrm{~m}$ long, with $0.2 \times 0.4 \mathrm{~m}$ rectangular section. One of the duct ends was terminated with noise generating loudspeaker, while the other was opened. The attenuating loudspeakers were of $0.16 \mathrm{~m}$ diameter and were situated approximately in the middle of the duct. The distance between the middles of the loudspeakers was equal to $0.3 \mathrm{~m}$. The reference microphone was located $1.23 \mathrm{~m}$ from the middle of L2a loudspeaker, while the error microphone was separated from L2b loudspeaker by the distance of $0.23 \mathrm{~m}$.

It should be emphasised that the goal of active noise control algorithm is in contradiction to the goal of the virtual unidirectional sound source adaptation algorithm [11]. The former tries to assure the compensation of sound waves from both the primary and secondary sources at the microphone M2 point without taking any notice of what happens at the microphone M1 point. The goal of the latter is to compensate sound waves from the secondary sources only at the microphone M1 point and try to assure the set point value appearing without any alternation at the microphone M2 point. This leads to a conclusion that both the algorithms should never operate at the same time. Indeed, the experiments show that when both the adaptation algorithms are in operation the whole system goes unstable. Therefore, the VUSS adaptation algorithm is usually run on the beginning of experiments and switched off after adaptation is completed. Next, only ANC algorithm is in use. Only when significant changes in the environment are detected (e.g., temperature change above some level), the ANC adaptation is temporarily frozen and the VUSS is retuned.

\section{FXLMS algorithm}

The first of ANC filter adaptation algorithms tested was Filtered- $x$ LMS algorithm $[4,9]$. Assume that the $F(z)$ is an FIR filter with coefficients (see Figure 4 ) in the ith sample of time stored in a form of a vector $\mathbf{f}(i)$. In that case, the FXLMS algorithm update equation is given by

$$
\mathbf{f}(i+1)=\mathbf{f}(i)+\mu \cdot \mathbf{x}^{\prime}(i) \cdot e(i),
$$

where $\mu$ is the step size and $\mathbf{x}^{\prime}(i)$ is the vector of reference signal $x(i)$ samples filtered by the secondary path transfer function estimate, that is, an estimate of the transfer function between set point value signal $t(i)$ and error signal $e(i)$.

If VUSS algorithm was working perfectly, the secondary path transfer function would be equal to simple time delay. Unfortunately, the study from Section 3.1 leads to a conclusion that such perfect situation is impossible: the
VUSS algorithm has to "pay more attention" to neutralising acoustic feedback and secondary path equalisation is only its additional task.

\section{RLS Algorithm}

The second algorithm of ANC filter adaptation algorithms tested was recursive least squares (RLS) algorithm (see, e.g., [9]). RLS algorithm uses very similar update equation in the form

$$
\mathbf{f}(i+1)=\mathbf{f}(i)+\mathbf{k}(i) \cdot e(i)
$$

where $\mathbf{k}(i)$ is the gain vector showing how much the value of $e(i)$ will modify different filter coefficients. The gain vector is calculated as

$$
\mathbf{k}(i)=\mathbf{P}(i) \cdot \mathbf{x}(i)
$$

where $\mathbf{P}(i)$ is a matrix updated in each step according to the following equation:

$$
\mathbf{P}(i)=\frac{1}{\lambda}\left(\mathbf{P}(i-1)-\frac{\mathbf{P}(i-1) \mathbf{x}(i-1) \mathbf{x}^{T}(i-1) \mathbf{P}(i-1)}{\lambda+\mathbf{x}^{T}(i-1) \mathbf{P}(i-1) \mathbf{x}(i-1)}\right) .
$$

The RLS algorithm usually converges faster than the LMS algorithm [12].

Because RLS algorithm needs to update $\mathbf{P}$ matrix of size equal to the number of filter coefficients in each adaptation step, it requires computational effort of $N^{2}$, whereas LMS algorithm requires computational effort of $N$ only [13]. There is, however, a family of RLS algorithm implementations called fast RLS algorithms that allows to omit computation of $\mathbf{P}$ matrix and use a selection of row vector instead $[13,14]$. This work used one of such fast algorithms, called fast transversal filter [15]. The algorithm was parametrised as follows. The initial value of the minimum sum of backward a posteriori prediction-error squares was equal to 1 and the exponential weighting factor was equal to 0.9999 . The algorithm showed no stability issues, as it was expected on floating-point arithmetic platform.

Both the FXLMS and RLS algorithms were implemented in $\mathrm{C}$ programming language. Testing and debugging were performed using developed simulation platform for Linux operating system. The platform allowed to emulate DSP processor board behaviour and therefore the next step, moving the code onto Texas Instruments TMS320C31 processor board, was purely automatic. Another PC-computer program was used to tune various algorithm parameters and to acquire the results. The sampling frequency of $2 \mathrm{kHz}$ was chosen as frequency band up to $1 \mathrm{kHz}$ was of the author interest. It appeared that, due to hardware limitations, appliable filter lengths were up to 70 with this sampling frequency.

\subsection{Testing sounds}

The set of signals chosen for experiments is presented with dashed line on Figures 12-17. Testing signals N1 and N2 
TABLE 1: The attenuation in simulations.

\begin{tabular}{lccc}
\hline Signal & Analytical solution + FXLMS & Analytical solution + FXLMS & Analytical solution + RLS \\
\hline N1 & $9 \mathrm{~dB}$ & $15 \mathrm{~dB}$ & $16 \mathrm{~dB}$ \\
N2 & $16 \mathrm{~dB}$ & $30 \mathrm{~dB}$ & $33 \mathrm{~dB}$ \\
N3 & $10 \mathrm{~dB}$ & $15 \mathrm{~dB}$ & $16 \mathrm{~dB}$ \\
N4 & $15 \mathrm{~dB}$ & $22 \mathrm{~dB}$ & $22 \mathrm{~dB}$ \\
N5 & $9 \mathrm{~dB}$ & $17 \mathrm{~dB}$ & $18 \mathrm{~dB}$ \\
N6 & $6 \mathrm{~dB}$ & $9 \mathrm{~dB}$ & $8 \mathrm{~dB}$ \\
\hline
\end{tabular}

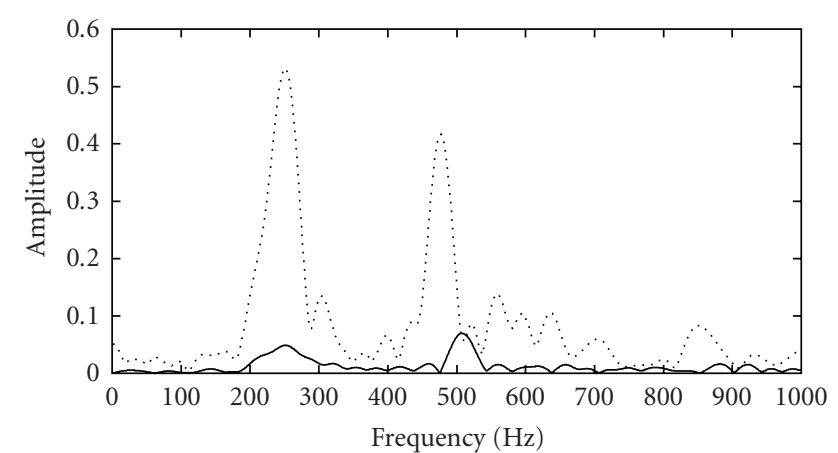

FIgURE 5: Amplitude spectrum of transfer function between set point value $t(i)$ and $x(i)$ (solid) and $e(i)$ (dotted).

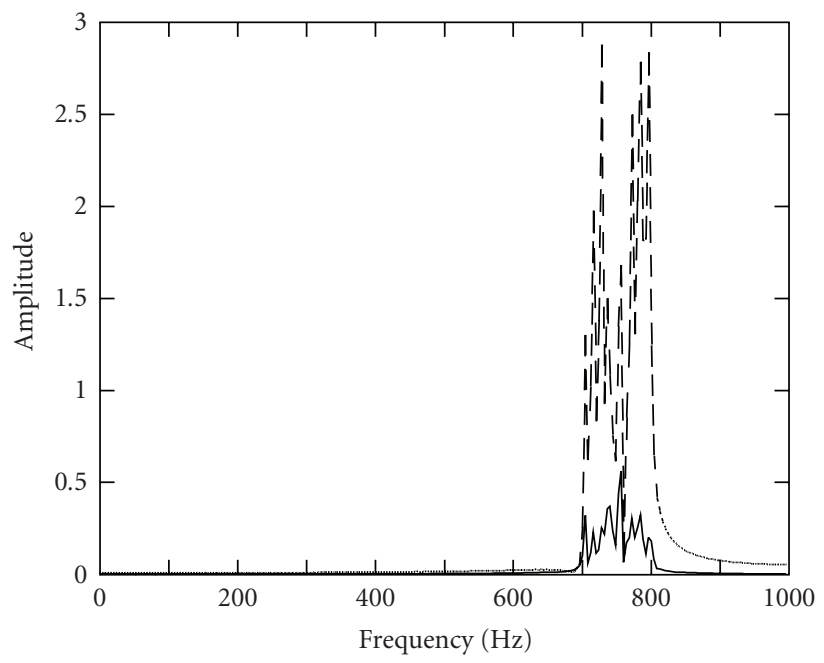

FIGURE 6: Noise N2 attenuation using analytical VUSS solution. Spectrum with ANC, - - - spectrum without ANC.

were generated offline as white noise filtered with highorder bandpass filter. Bandpass was $200-300 \mathrm{~Hz}$ in case of N1 signal and $700-800 \mathrm{~Hz}$ in case of signal N2. The former was chosen to show low-frequency attenuation capabilities and the latter to show high frequency attenuation. The N3 is a signal acquired in close vicinity of a food processor. It has the dominant frequency of about $270 \mathrm{~Hz}$, but with substantial amount of broadband noise. The N4 is a signal collected near a power transformer. It has all the harmonics of $50 \mathrm{~Hz}$ visible

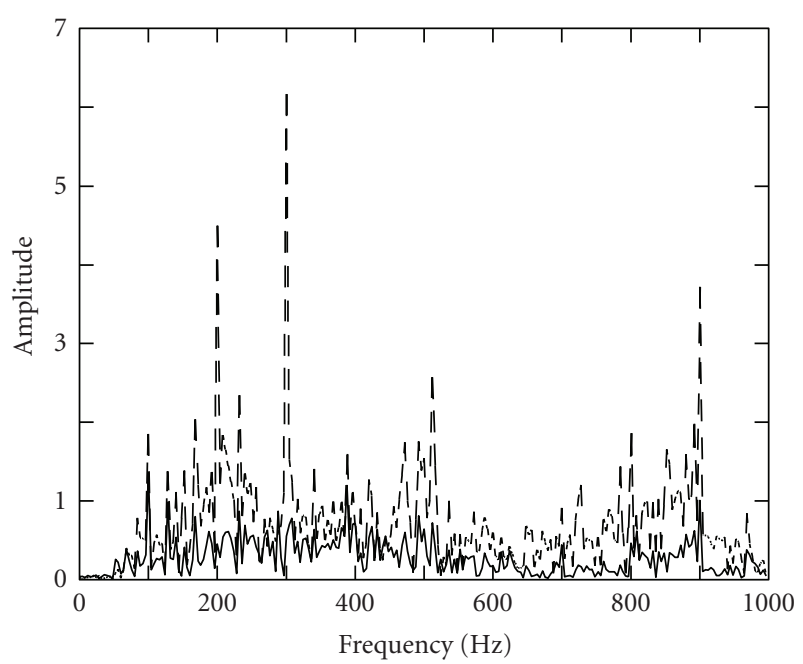

FIGURE 7: Noise N5 attenuation using analytical VUSS solution. Spectrum with ANC, - - - spectrum without ANC.

with $250 \mathrm{~Hz}$ being the dominant. The N5 signal was recorded in water power plant turbine proximity. It has the harmonics of $100 \mathrm{~Hz}$ distinguishable among broadband noise. The last signal N6 was recorded in small bureau with an electric propeller fan turned on. It is similar to N5, but the dominant frequency is higher.

All the signals were filed using 1000 samples. This ensemble was repeated many times and (after amplification) sent to loudspeaker L2 (see Figure 4). The results of attenuation were measured with microphone M2 and calculated as

$$
\mathcal{T}=10 \log \left(\frac{\text { MSV of } e(i) \text { without attenuation }}{\operatorname{MSV} \text { of } e(i) \text { with attenuation }}\right)[\mathrm{dB}] .
$$

\subsection{Simulation results}

The simulations were performed using 150th order FIR filters models of the duct paths, identified offline using methodology presented in [16]. The algorithm for suboptimal VUSS filter solution described in Section 3.1 was tested first. As expected, it allowed for very effective acoustical feedback cancellation but at the cost of the secondary path being substantially degenerated. The amplitude spectrum of transfer function between set point value $t(i)$ and both the reference signal $x(i)$ and error signal $e(i)$ is presented on Figure 5. 


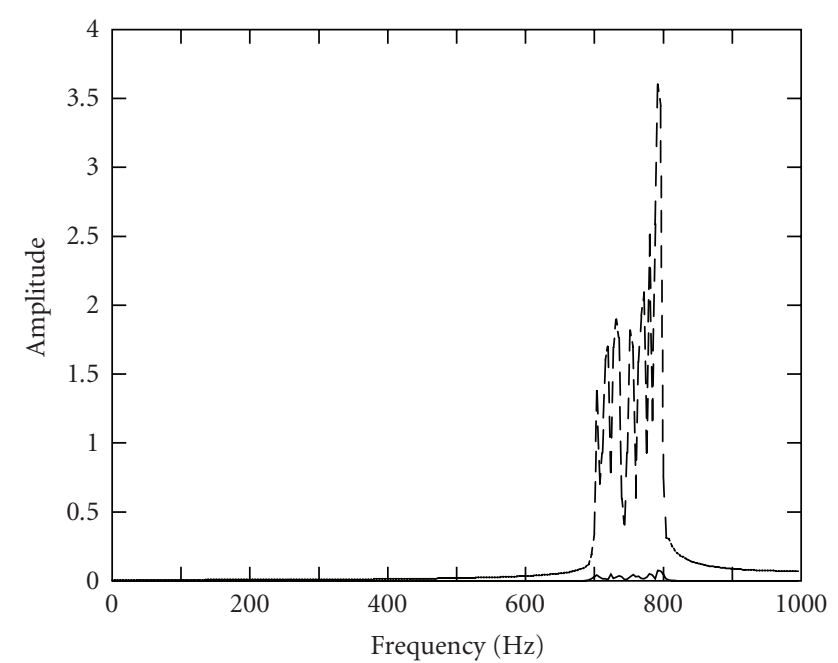

FIgURE 8: Noise N2 attenuation using adaptive VUSS solution. Spectrum with ANC, - - - spectrum without ANC.

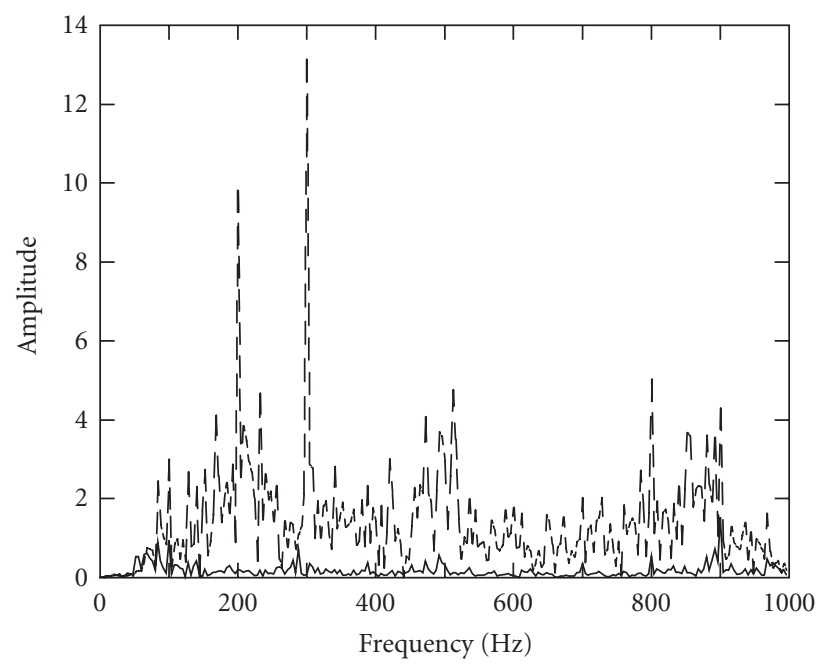

FIgURE 9: Noise N5 attenuation using adaptive VUSS solution. Spectrum with ANC, - - - spectrum without ANC.

After tuning phase, the system was engaged to active noise control algorithms: FXLMS and Fast RLS. The ANC filter having 70 coefficients proved to be long enough to cancel out the noise in case of tonal sounds, so this was the chosen value. However, in case of N1-N6 signals attenuation was only (as for simulations of feedforward controller) 6$16 \mathrm{~dB}$ (with FXLMS algorithm, which proved to be better), see Table 1 . The examples of spectra of the signals observed at the error microphone before and after attenuation have been presented on Figures 6 and 7 .

In the following experiments, adaptive algorithm described in Section 3.2 was responsible for VUSS filters tuning. The value of the $\beta$ parameter (see (45)) has been chosen as 0.5 to put the same effort into satisfying both the goals defined by (12). The attenuation of tonal sounds

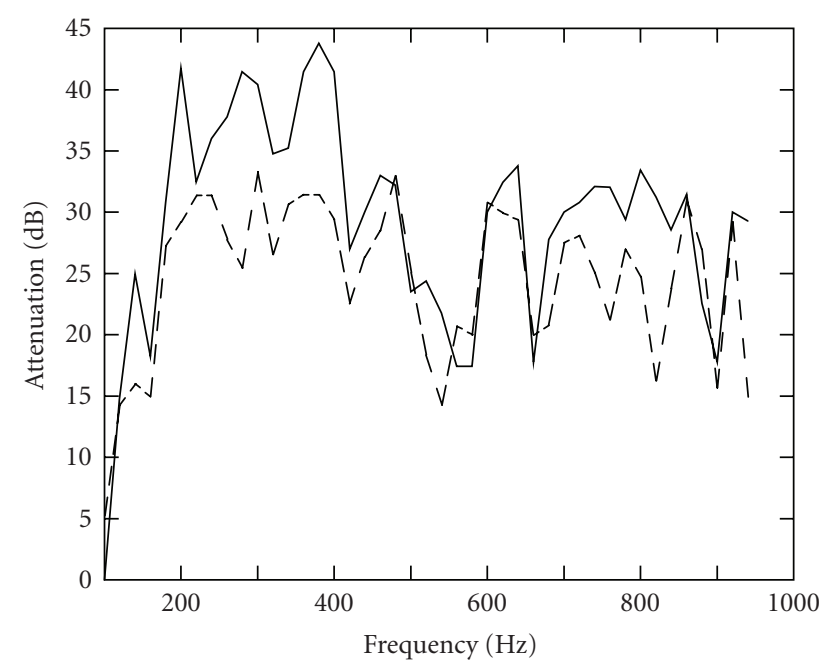

Figure 10: Attenuation (in $\mathrm{dB}$ ) of tonal sounds for adaptive filter tuning. - RLS algorithm, - - - FXLMS algorithm.

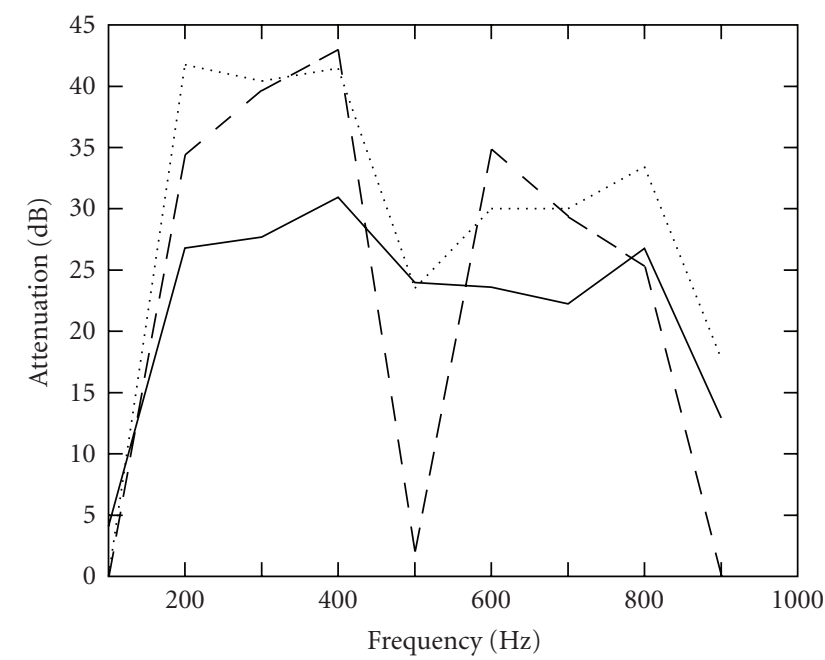

FIGURE 11: Comparison of attenuation of tonal sounds for analytical and adaptive VUSS filter tuning. - FXLMS algorithm with analytical tuning, - - - RLS algorithm with analytical tuning, ... RLS algorithm with adaptive tuning.

was also infinite in case of adaptive VUSS filters tuning. But the attenuation of testing signals was slightly better: 9-30 dB in case of FXLMS algorithm and $8-33 \mathrm{~dB}$ in case of RLS algorithm, see Table 1 . The examples of spectra of the signals observed at the error microphone before and after attenuation have been shown on Figures 8 and 9. The difference in amplitude spectrum of the N2 and N5 signals without attenuation visible on these figures and on Figure 6 and 7 comes from the fact that this is the spectrum measured with the M2 microphone, not the spectrum of the signal driving the L1 loudspeaker.

Table 1 summarises the results obtained during simulations for broadband signals N1-N6. The simulation experiments proved adaptive VUSS filters tuning superiority over analytical suboptimal filter design. 


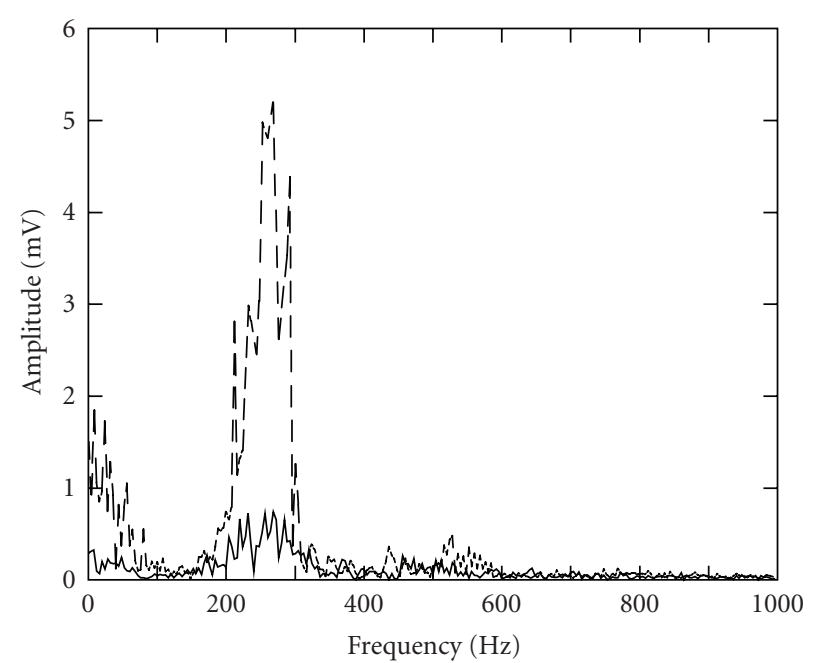

Figure 12: Noise N1 (broadband noise 200-300 Hz) attenuation. - Spectrum with ANC, - - spectrum without ANC.

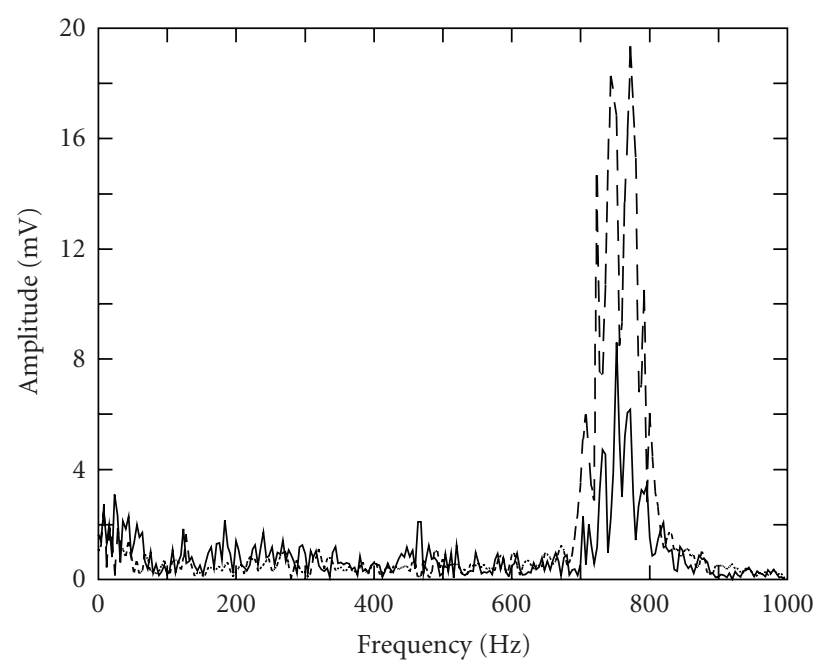

FIgURE 13: Noise N2 (broadband noise 700-800 Hz) attenuation. - Spectrum with ANC, - - - spectrum without ANC.

\subsection{Real experiments}

To perform the real experiments using analytical filter design it was necessary to identify the models of electroacoustic paths $S_{11}(z)-S_{22}(z)$ (see Figure 3 ) first. The identification of FIR models of these paths was performed using LMS algorithm prior to each experiment. Moreover, in case of both the analytical and adaptive filter designs it was necessary to identify the model of the secondary path before the ANC. The identification of FIR model of this path was performed after VUSS was tuned by online identification procedure. The procedure, however, was disabled during the ANC.

The first ANC laboratory experiments used tonal sounds. The frequency range between 100 and $950 \mathrm{~Hz}$ has been checked with resolution of $20 \mathrm{~Hz}$. The VUSS filter design was performed using adaptive method. The attenuation obtained during these experiments has been shown on Figure 10. The

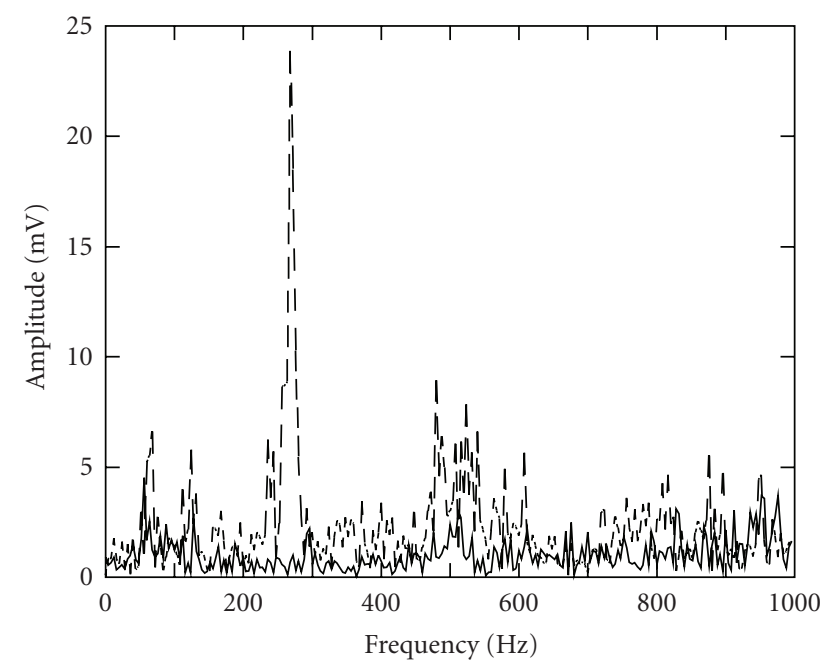

FIgUre 14: Noise N3 (food processor) attenuation. - Spectrum with ANC, - - - spectrum without ANC.

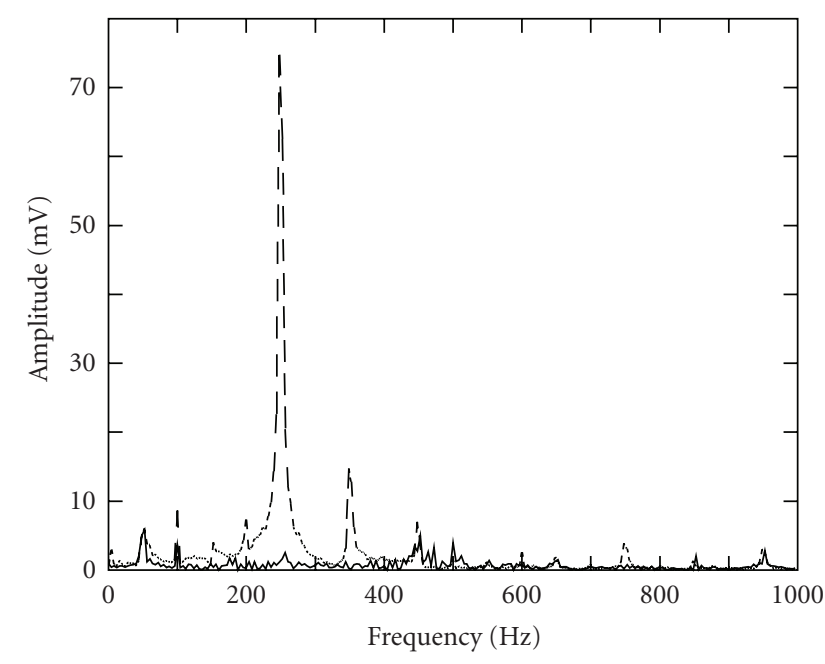

FIgURE 15: Noise N4 (power transformer) attenuation. - Spectrum with ANC, - - - spectrum without ANC.

figure shows that in frequency range from 150 to $950 \mathrm{~Hz}$ the attenuation was between 14 and $33 \mathrm{~dB}$ (average $25 \mathrm{~dB}$ ) for FXLMS ANC algorithm and between 15 and $44 \mathrm{~dB}$ (average $30 \mathrm{~dB}$ ) for RLS ANC algorithm.

The drop in attenuation above a frequency of about $420 \mathrm{~Hz}$, especially distinct in case of RLS algorithm, can be explained as the ducts first cut-on frequency appears at $425 \mathrm{~Hz}$. The figure shows that the performances of both FXLMS and RLS algorithms above first cut-on frequency are similar. However, below a frequency of $400 \mathrm{~Hz}$ the performance of RLS algorithm is up to $14 \mathrm{~dB}$ better than in case of FXLMS algorithm.

The next step was to compare the performance of ANC system in case of analytical and adaptive filter design. During these experiments frequency range from 100 to $900 \mathrm{~Hz}$ was tested with resolution of $100 \mathrm{~Hz}$. The results are shown on Figure 11. In all cases the efficiency of ANC algorithm with 
TABLE 2: The attenuation in real experiments.

\begin{tabular}{lcc}
\hline Signal & Adaptive solution + FXLMS & Adaptive solution + RLS \\
\hline N1 & $12 \mathrm{~dB}$ & $14 \mathrm{~dB}$ \\
N2 & $7 \mathrm{~dB}$ & $7 \mathrm{~dB}$ \\
N3 & $4 \mathrm{~dB}$ & $8 \mathrm{~dB}$ \\
N4 & $13 \mathrm{~dB}$ & $16 \mathrm{~dB}$ \\
N5 & $3 \mathrm{~dB}$ & $8 \mathrm{~dB}$ \\
N6 & $3 \mathrm{~dB}$ & $5 \mathrm{~dB}$ \\
\hline
\end{tabular}

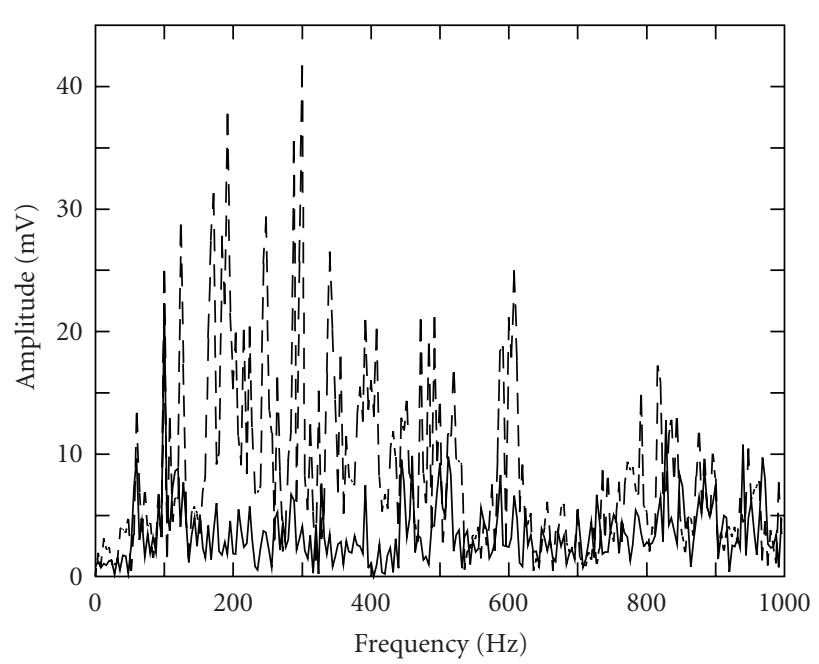

FIgURE 16: Noise N5 (power plant turbine) attenuation. Spectrum with ANC, - - - spectrum without ANC.

analytical VUSS tuning was worse than efficiency of the same algorithm with adaptive VUSS tuning. Therefore, only adaptive VUSS design was considered for the following tests.

Finally, the noise signals described in Section 4.2 were used in real experiments. Although both FXLMS and RLS ANC algorithms were checked for performance, the only spectra presented on Figure 12 through Figure 17 are those obtained with RLS algorithm, as this algorithm performance was superior to FXLMS in all experiments. The attenuation factors for both FXLMS and RLS algorithms are presented in Table 2.

\section{CONCLUSIONS}

The idea of virtual unidirectional sound source presented in this paper is based on theoretical study of wave propagation in duct. VUSS itself is a special case of two-reference, twooutput system with a detailed analysis presented in Section 3. Its application to active noise control in an acoustic duct proved to be effective, resulting in $20-40 \mathrm{~dB}$ attenuation of tonal sounds and $5-16 \mathrm{~dB}$ attenuation of complex signals with broadband noise. The best results were obtained with adaptive VUSS design together with RLS active noise control algorithm. These results are comparable with similar results reported by other authors. The results, however, do not show

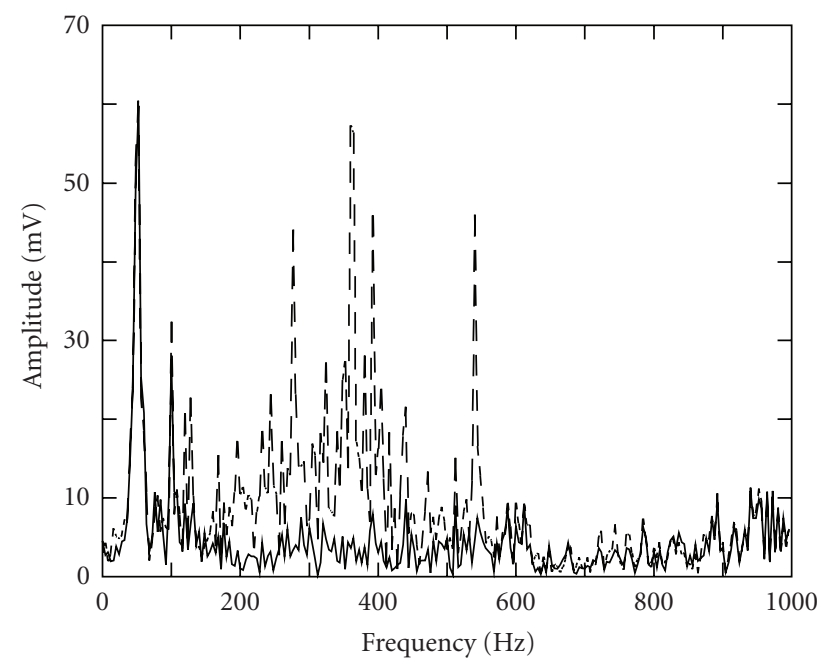

Figure 17: Noise N6 (electric propeller fan) attenuation. Spectrum with ANC, - - - spectrum without ANC.

one of the strengths of VUSS, the ability to adapt to time varying feedback paths.

\section{REFERENCES}

[1] D. Bismor, "Różnice pomidzy obiektami automatyki przemysłowej a obiektami akustycznymi w świetle doświadczeń identyfikacyjnych," in Materiały XXV Zimowej Szkoly Zwalczania Zagrożeń Wibroakustycznych, Gliwice-Ustroń, Poland, 1997.

[2] M. T. Akhtar, M. Abe, and M. Kawamata, "On active noise control systems with online acoustic feedback path modeling," IEEE Transactions on Audio, Speech and Language Processing, vol. 15, no. 2, pp. 593-600, 2007.

[3] S. Elliott, Signal Processing for Active Noise Control, Academic Press, London, UK, 2001.

[4] S. Kuo and D. Morgan, Active Noise Control Systems, John Wiley \& Sons, New York, NY, USA, 1996.

[5] J. Poshtan, S. Sadeghi, and M. H. Kahaei, "An investigation on the effect of acoustic feedback in a single-channel active noise control system," in Proceedings of the IEEE Conference on Control Applications (CCA '03), vol. 1, pp. 430-434, Istanbul, Turkey, June 2003.

[6] T. Habib, M. Akhtar, and M. Arif, "Acoustic feedback path modeling and neutralization in active noise control systems," in Proceedings of the IEEE Multitopic Conference (INMIC '06), pp. 89-93, Islamabad, Pakistan, December 2006. 
[7] V. Välimäki and S. Uosukainen, "Adaptive design of a unidirectional source in a duct," in Proceedings of the 23th International Conference on Noise and Vibration Engineering (ISMA23 '98), vol. 3, pp. 1253-1260, Leuven, Belgium, September 1998.

[8] E. Wong, Procesy Stochastyczne w Teorii Informacji i Układów Dynamicznych, WNT, Warszawa, Poland, 1976.

[9] S. Haykin, Adaptive Filter Theory, Prentice-Hall, New York, NY, USA, 4th edition, 2002.

[10] A. K. Wang and W. Ren, "Convergence analysis of the multivariable filtered-X LMS algorithm with application to active noise control," IEEE Transactions on Signal Processing, vol. 47, no. 4, pp. 1166-1169, 1999.

[11] D. Bismor, "Generation of effect of virtual undirectional source of sound using adaptive techniques," Archives of Control Sciences, vol. 13, no. 2, pp. 215-230, 2003.

[12] D. Bismor, "RLS algorithm in active noise control," in Proceedings of the 6th International Congress on Sound and Vibration (ICSV'99), Lyngby, Denmark, July 1999.

[13] A. H. Sayed, Fundamentals of Adaptive Filtering, John Wiley \& Sons, New York, NY, USA, 2003.

[14] L. Rutkowski, Filtry Adaptacyjne i Adaptacyjne Przetwarzanie Sygnałów, WNT, Warszawa, Poland, 1994.

[15] J. Cioffi and T. Kailath, "Fast, recursive-least-squares transversal filters for adaptive filtering," IEEE Transactions on Acoustics, Speech and Signal Processing, vol. 32, no. 2, pp. 304-337, 1984.

[16] D. Bismor, Adaptive Algorithms for Active Noise Control in an Acoustic Duct, Jacek Skalmierski Computer Studio, Gliwice, Poland, 1999. 

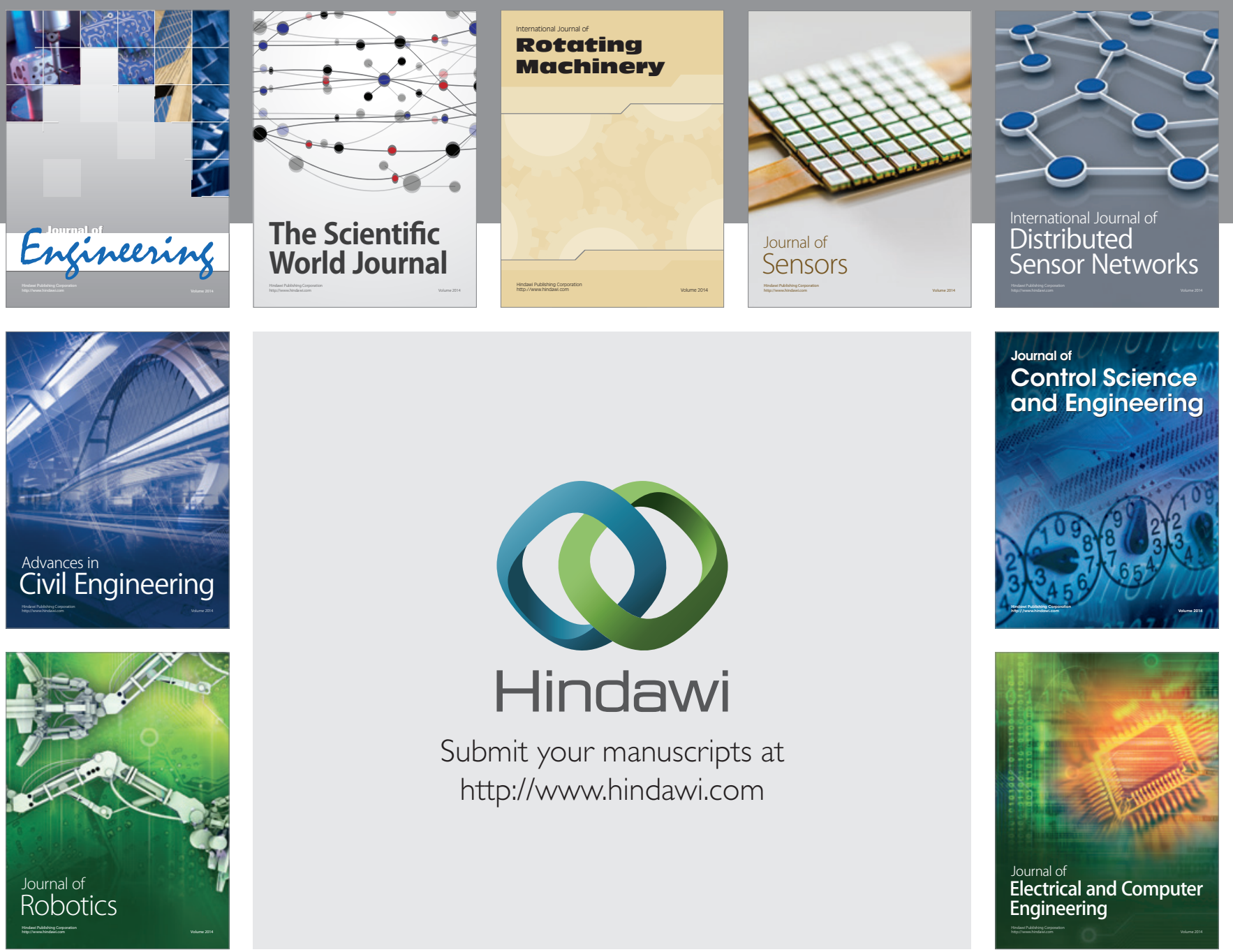

Submit your manuscripts at

http://www.hindawi.com
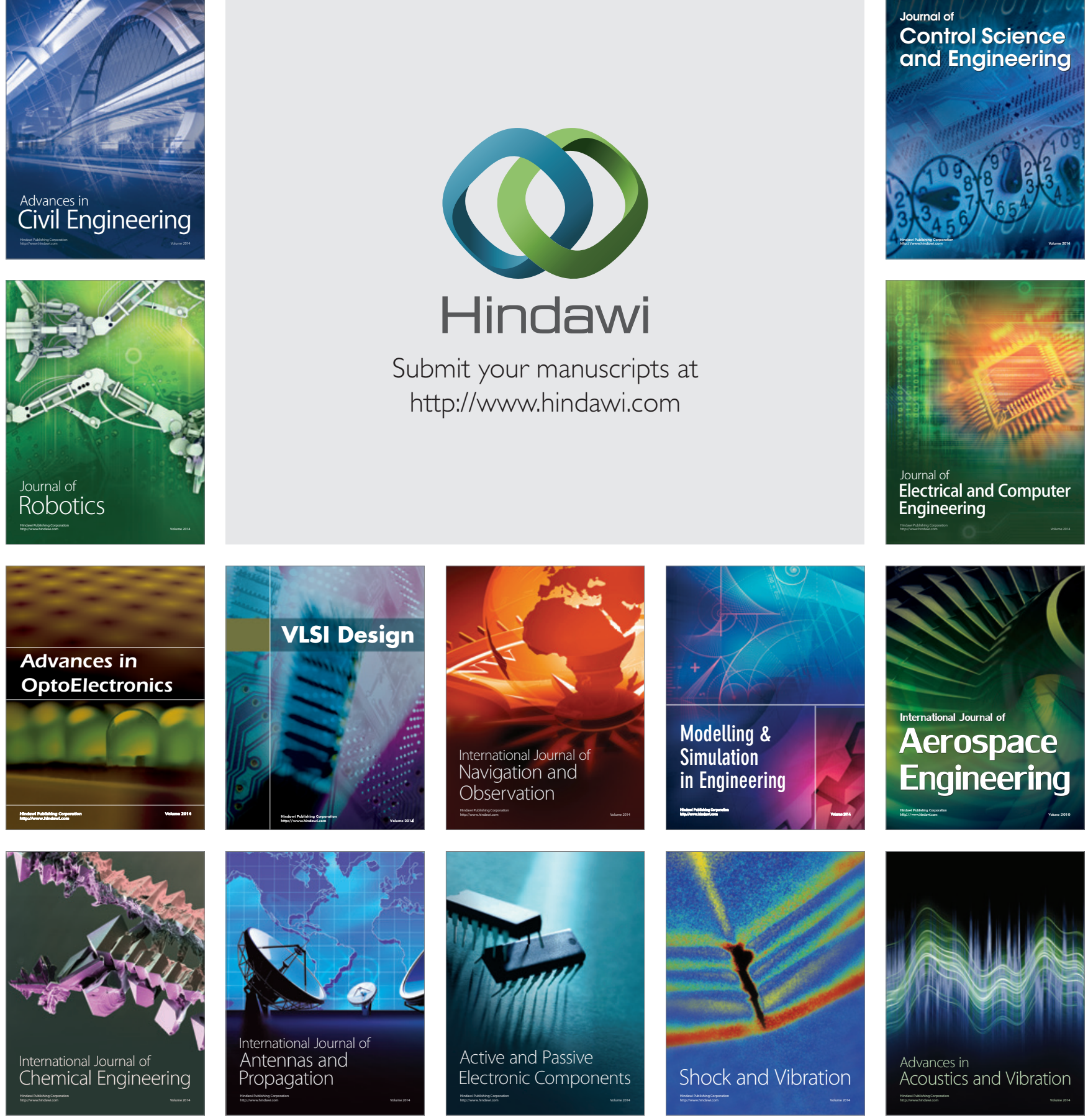